\title{
Rare Diagnosis of Krukenberg Tumor: Intrahepatic Cholangiocarcinoma
}

\author{
Tiago Castello Branco Lyra1, Fernando Morbeck ${ }^{1}$, Marcos Duarte Guimarães ${ }^{1,2}$, \\ Luiz Felipe Sias Franco', Edson Marchiori ${ }^{3}$, Glaucio Siqueira1, Carlos Chaves Faloppa², \\ Felipe D'almeida Costa ${ }^{2}$, Stephania Martins Bezerra ${ }^{2}$ \\ ${ }^{1}$ Department of Radiology, Hospital Heliopolis, Sao Paulo, Brazil \\ ${ }^{2}$ AC Camargo Cancer Center, Sao Paulo, Brazil \\ ${ }^{3}$ Universidade Federal do Rio de Janeiro, Rio de Janeiro, Brazil \\ Email: tiagocbl@hotmail.com
}

Received 19 July 2015; accepted 26 September 2015; published 29 September 2015

Copyright (C) 2015 by authors and Scientific Research Publishing Inc.

This work is licensed under the Creative Commons Attribution International License (CC BY). http://creativecommons.org/licenses/by/4.0/

c) (†) Open Access

\begin{abstract}
Krukenberg tumor is a rare ovarian metastatic cancer from the gastrointestinal tract. It accounts for $1 \%-5 \%$ of malignant tumors of the ovaries, usually being bilateral, of solid consistency and may have cystic areas. The World Health Organization (WHO) defined it as an ovarian metastasis when the mucus-secreting cells of "signet ring" are present, associated with sarcomatous stromal proliferation. We present a 58-year-old woman with the diagnosis of peripheric cholangiocarcinoma with ovarian metastasis, showing history of pain and increased abdominal volume. Magnetic Resonance Imaging (MRI) showed multiple hepatic solid nodules with predominantly peripheral, centripetal and progressive enhancement, demonstrating hipersignal and restriction on diffusion weighted imaging (DWI). In the anexial region, bulky heterogeneous multiloculated cystic formations with solid components, of probable ovarian origin, were noted. Biopsy of the liver nodules confirmed the diagnosis of cholangiocarcinoma.
\end{abstract}

\section{Keywords}

Krukenberg, Cholangiocarcinoma, Tumor, MRI

\section{Introduction}

Cholangiocarcinoma is a rare tumor of the biliary tract, despite being the second most common primary neoplasm of the liver. It is associated with poor prognosis and high morbidity levels, even after curative intention. A Krukenberg tumor refers to a malignancy in the ovary metastasized from a primary site, classically the gastroin- 
testinal tract, although it can arise in other tissues such as the breast. A Krukenberg tumor refers to a malignancy in the ovary that is metastasized from a primary site, classically the gastrointestinal tract: up to $70 \%$ to $90 \%$ of Krukenberg tumors originate from gastric mucosa; 14\% from the colon mucosa; 5\% of Gall Bladder and Pancreas, and $2 \%$ has appendicular origin, although it can arise in other tissues such as the breast [1] [2]. It can be seen in all age groups, with an average age of 45 years. Krukenberg tumors often come to the attention when they cause abdominal or pelvic pain, bloating, ascites, or pain during sexual intercourse [3]. It can occasionally induce a reaction of the ovarian stroma which leads to hormone production, resulting in vaginal bleeding, a change in menstrual habits or occasionally hirsutism as a main symptom. All these symptoms are non-specific and can also arise with a range of problems other than cancer, and a diagnosis can only be made following confirmatory investigations such as computed tomography (CT) scans and/or laparotomy [3] [4].

We report a patient with the diagnosis of intrahepatic cholangiocarcinoma, with ovarian metastasis (Krukenberg tumor). It's important to contemplate this diagnosis in women with ovarian mass of unknown origin, despite your rarity.

\section{Case Presentation}

A 58-year-old woman, housewife, born and living in São Paulo, with a history of pain and increased abdominal volume associated with early vaginal bleeding and change in bowel pattern, without hematochezia. There was also some weight loss. On physical examination, it was noted distended abdomen, ascites, and palpable masses on both flanks. In laboratory tests, Gamma-GT levels were 116 U/L, AST 46 U/L, ALT 40 U/L, Alkaline phosphatase $138 \mathrm{U} / \mathrm{L}$ and LDH $490 \mathrm{U} / \mathrm{L}$.

Ultrasonography (US) showed bilateral pelvic expansive formations and also focal hepatic lesions, continuing to research with MRI exam. The following findings stood out: lobulated liver, preserved dimensions, noting solid nodules, lobulated, sometimes confluent, presenting predominantly peripheral enhancement by contrast medium, with centripetal and progressive impregnation (Figure 1, Figure 2), associated also to the restriction on diffusion (Figure 3 and Figure 4), measuring up to $48 \times 40 \mathrm{~mm}$ in the largest axial shafts; ascites and peritoneal implants (Figure 5). There was expansion of intra and extrahepatic bile ducts.

In the pelvic area, were noted bulky cystic formations, multiloculated associated with the solid component of well-defined contours, with heterogeneous enhancement by contrast medium, occupying much of the pelvic excavation, probably from ovarian origin (Figure 6, Figure 7).

The main hypothesis was intrahepatic cholangiocarcinoma (peripheral) with metastatic spread to the ovaries. Biopsy of the liver nodules, which resulted in adenocarcinoma. The immunohistochemical study of the profile showed the diagnosis of cholangiocarcinoma (Figure 8).

\section{Discussion}

Intrahepatic cholangiocarcinoma is the second most common primary malignancy of the liver ( $10 \%$ to $15 \%$ of

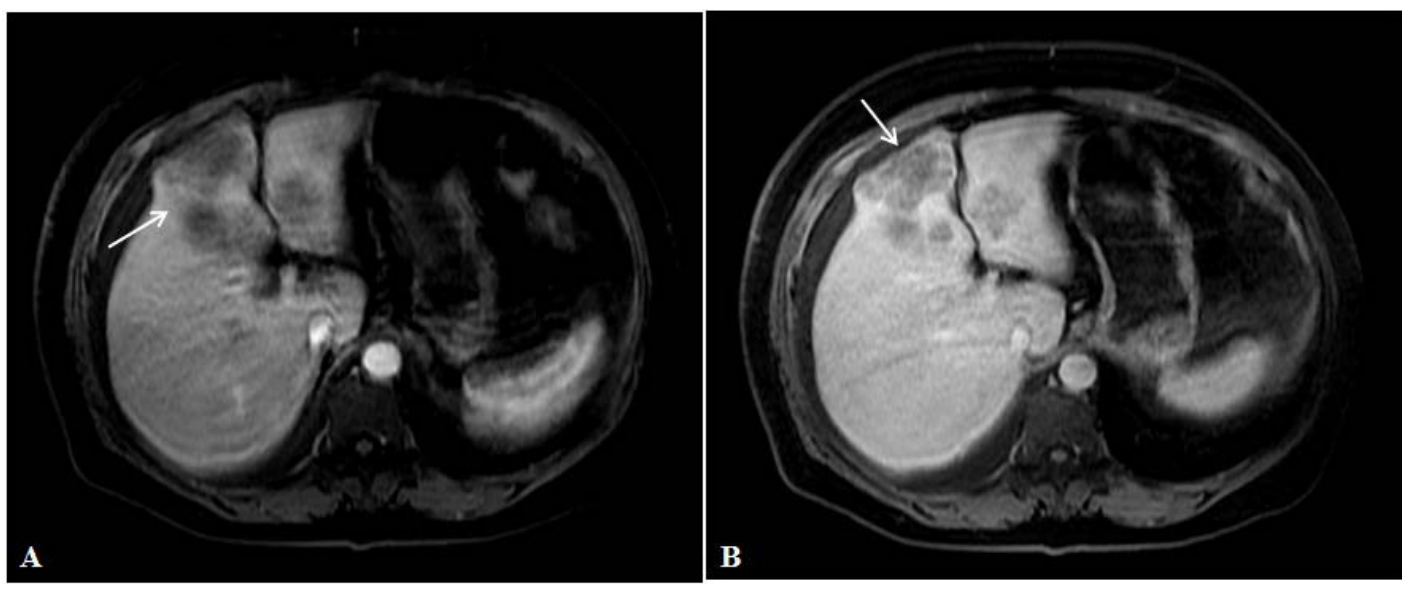

Figure 1. MRI T1 axial plan after injection of contrast in the arterial (A) and late (B) phases, demonstrating solid nodular lesion with irregular, centripetal and progressive enhancement (white arrows). 

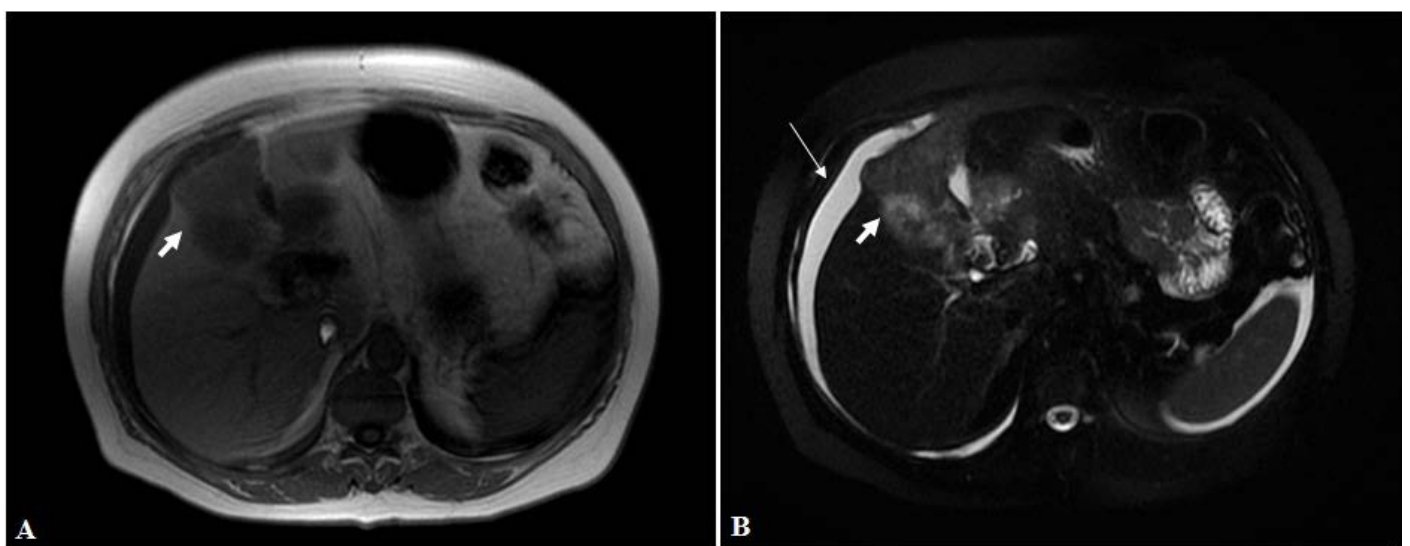

Figure 2. (A) MRI T1 axial plan demonstrating nodules hipointenses in the segment IV and (B) MRI T2 axial plan demonstrating nodules solids hyperintenses in this acquisition (thick white arrows). Ascites is well demonstrated in (B) (thin white arrow).
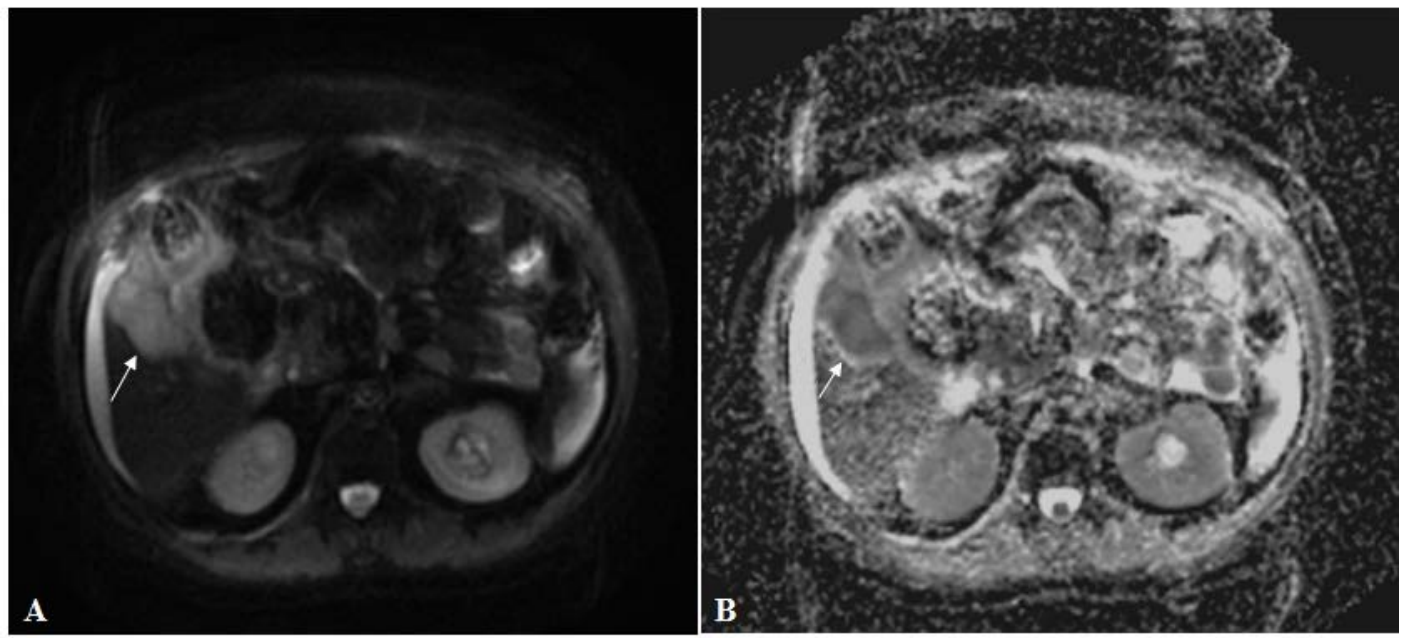

Figure 3. (A) MRI diffusion sequence in axial plan with b value of 200 demonstrating hiperintense hepatic nodule in the segment VI; (B) MRI apparent diffusion coefficient (ADC) acquisition demonstrating the same nodule with hiposignal in this sequence, confirming restriction of water molecules diffusion (white arrows).

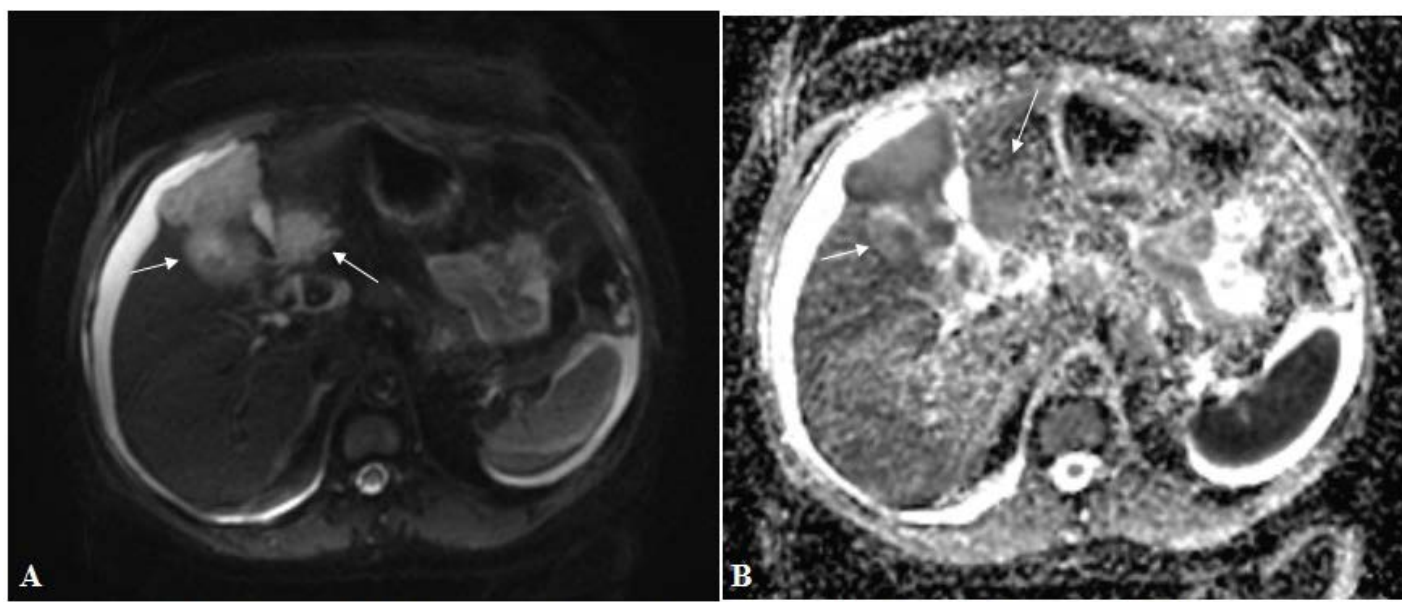

Figure 4. (A) MRI DWI axial plan showing nodular lesion with hyperintensity in the segments II and IV of the liver (white arrows); (B) MRI diffusion axial plan. 


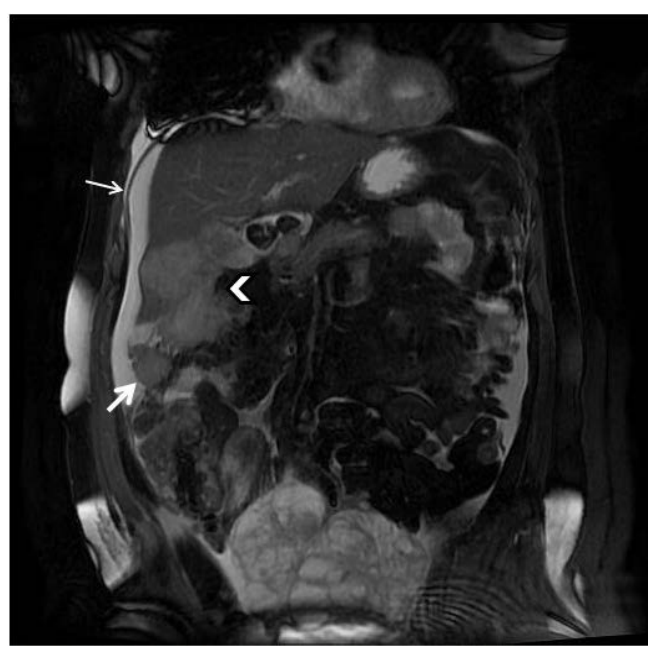

Figure 5. MRI Fiesta coronal plan showing ascites (thin white arrow), hepatic nodules (head white arrow) and peritoneal implants (thick white arrow).

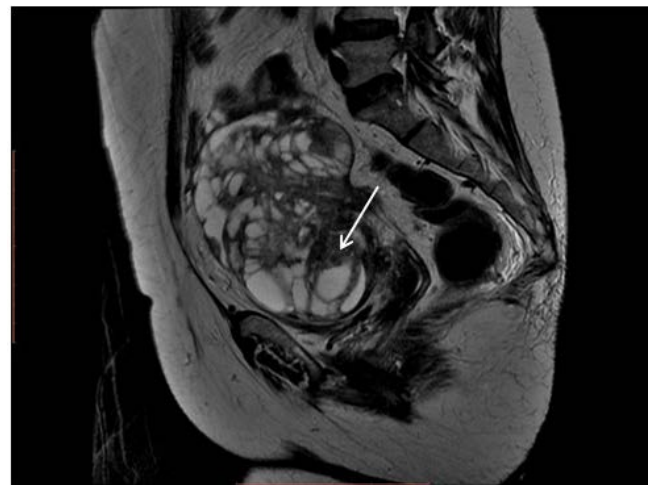

Figure 6. MRI T2 sagital plan showing cystic formation multiloculated with solid component areas (white arrow).

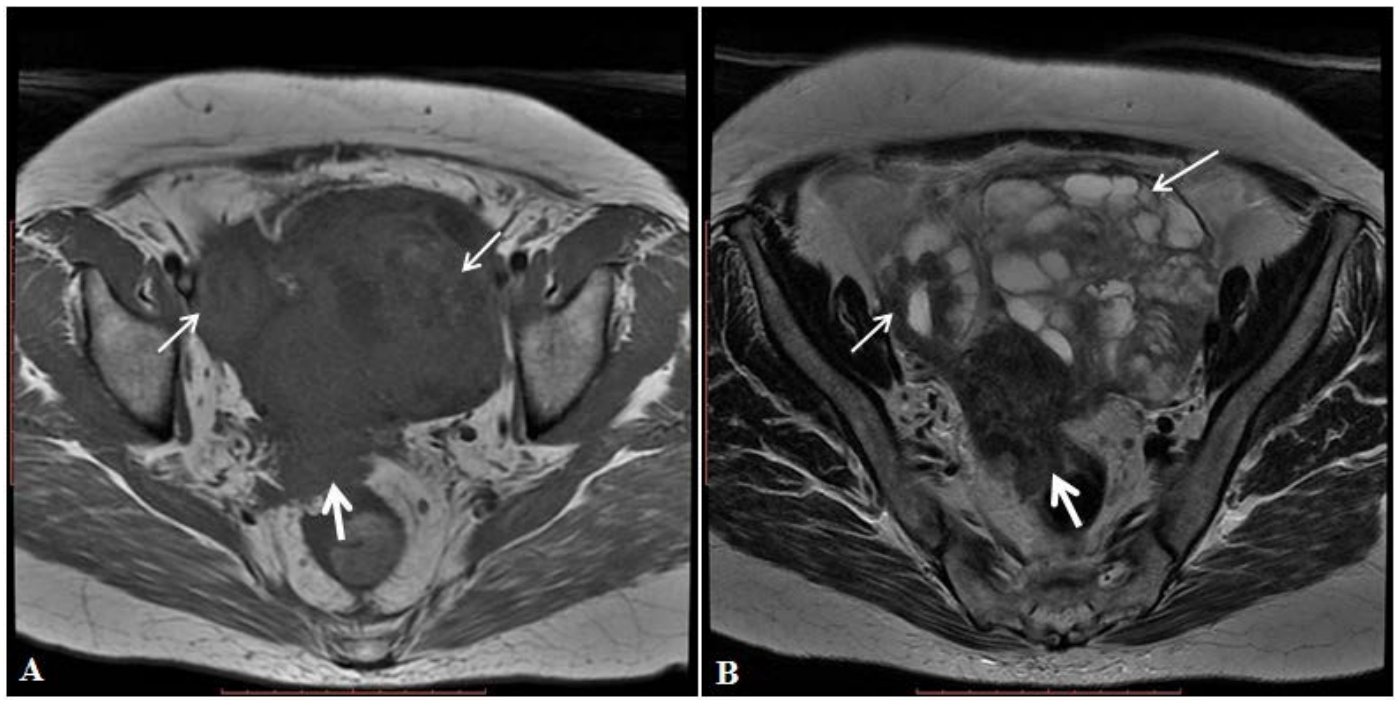

Figure 7. (A) MRI T1 axial plan and (B) MRI T2 axial plan. Note the anexial (thin arrows) and retrouterine (thick arrow) implants. 


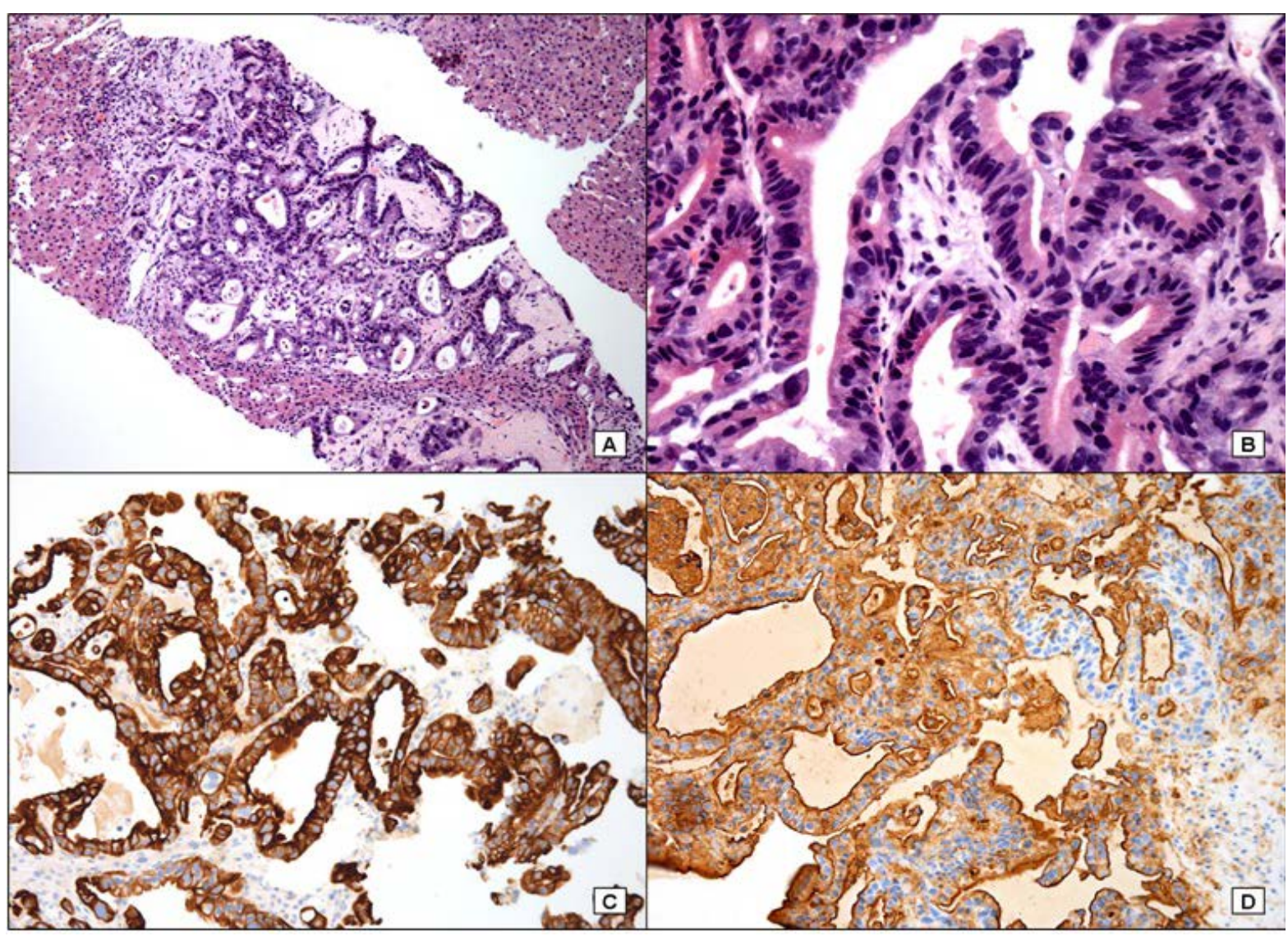

Figure 8. (A) Biopsy demonstrating irregular glands infiltrating fragments of neoplasic liver parenchyma (H\&E, 100×); (B) In a greater increase, glands show pacreato-biliary standard with atypical columnar cells with eosinophilic cytoplasm (H\&E, 400×); (C) (D) These cells showed positivity for cytokeratin 7 (immunohistochemistry with CK7 anti-antibody, 200×), and CA 19.9 (immunohistochemistry with CA 19.9 anti-antibody, 200×), confirming the diagnosis of cholangiocarcinoma.

primary liver tumors). It is more common in males, after 70 years [5] [6]. Its main risk factors are primary sclerosing cholangitis, choledochal cyst, steatohepatitis, cirrhosis and hepatitis B and C. Most common metastatic dissemination sites are the liver, lungs, adrenal glands and bones. In about $20 \%$ of the cases, there is peritoneal spread, including to the ovaries. Only $5 \%$ of Krukenberg tumors has its primary origin in the Gallbladder [1] [2] [7].

Usually it has nonspecific presentation in the initial phase: weight loss, pain or palpable mass. Can be asymptomatic in $30 \%$ of cases, being detected as an incidental mass on imaging exams [6].

The intrahepatic cholangiocarcinoma is classified into three types: bulk-forming (most common), intraductal growth and periductal infiltrative.

The bulk-forming type is usually seen on ultrasound as a homogeneous mass of intermediate echogenicity, with a peripheral hypoechoic halo [2] [5]. Already computed tomography (CT) and MRI are the methods of choice for staging and therapeutic planning of the injury. On CT, is presented as hypoattenuating mass in precontrast phase, while in the RM, usually there is a low signal on T1-weighted sequences and variable signal in T2, often displaying central hypointense (fibrosis) and may or may not be related dilatation of the bile ducts. In both imaging methods, the injury has similar behavior to the contrast with peripheral irregular enhancement with progressive and preferably late centripetal impregnation. Generally, satellites lesions may be associated with nodules and vascular involvement without the formation of coarse tumor thrombus. Another common imaging finding is hepatolithiasis and obliteration of the portal vein, conditioning atrophy of the involved segment.

Metastatic ovarian tumors are usually secondary to gastrointestinal neoplasms, followed breast, lung and contralateral ovaries [8] [9]. They account for about $5 \%-10 \%$ of all ovarian tumors, typically being bilateral. Image spectrum is quite wide, more commonly shown as complex bilateral lesions with solid and cystic areas and can 
be seen hyperintense on T1 and T2 (mucin) inside. Such findings often make it difficult to differentiate from primary neoplasms [3] [5]. Our case occurred in a 58 years old woman without risk factors. He presented with nonspecific clinical weight loss, vaginal bleeding and palpable mass. The image findings raised suspected and biopsy confirmed the diagnosis.

\section{Conclusions}

The diagnosis of Krukenberg tumor still remains a challenge for the radiologist and the surgeon, but should not be forgotten in the follow-up of female patients with history of adenocarcinoma, especially if it has gastrointestinal origin.

Therefore, we must always consider the diagnosis of metastatic disease to find solid ovarian lesions, especially if there is a history of a primary tumor, in particular from gastrointestinal tract, as the cholangiocarcinoma.

\section{Conflict Interest}

There is no conflict of interest.

\section{References}

[1] Chen, M.F. (1999) Peripheral Cholangiocarcinoma (Cholangiocellular Carcinoma): Clinical Features, Diagnosis and Treatment. Journal of Gastroenterology and Hepatology, 14, 1144-1149. http://dx.doi.org/10.1046/j.1440-1746.1999.01983.x

[2] Abou-Alfa, G.K., Jarnagin, W., Lowery, M., et al. (2014) Liver and Bile Duct Cancer. In: Niederhuber, J.E., Armitage, J.O., Doroshow, J.H., Kastan, M.B. and Tepper, J.E., Eds., Abeloff's Clinical Oncology, 5th Edition, Sanders, Philadelphia, 1373-1396.

[3] Fox, H. (1985) Metastatic Tumours of the Ovary. In: Haynes, M., Taylor, C. and Fox, H., Eds., Obstetrical and Gynecological Pathology, 3rd Edition, Edinburg, Livingstone, 714-723.

[4] Kim, H.K., Heo, D.S., Bang, Y.J. and Kim, N.K. (2001) Prognostic Factors of Krukenberg’s Tumor. Gynecologic Oncology, 82, 105-109. http://dx.doi.org/10.1006/gyno.2001.6210

[5] Chung, Y.E., et al. (2009) Varying Appearances of Cholangiocarcinoma: Radiologic-Pathologic Correlation. Radiographics, 29, 683-700. http://dx.doi.org/10.1148/rg.293085729

[6] Han, J.K., et al. (2002) Cholangiocarcinoma: Pictorial Essay of CT and Cholangiographic Findings. Radiographics, 22, 173-187. http://dx.doi.org/10.1148/radiographics.22.1.g02ja15173

[7] Jung, S.E., et al. (2002) CT and MR Imaging of Ovarian Tumors with Emphasis on Differential Diagnosis. Radiographics, 22, 1305-1325. http://dx.doi.org/10.1148/rg.226025033

[8] Seidman, J.D., Kurman, R.J. and Ronnett, B.M. (2003) Primary and Metastatic Mucinous Adenocarcinomas in the Ovaries: Incidence in Routine Practice with a New Approach to Improve Intraoperative Diagnosis. American Journal of Surgical Pathology, 27, 985-993. http://dx.doi.org/10.1097/00000478-200307000-00014

[9] Moore, R.G., Chung, M., Granai, C.O., Gajewski, W. and Steinhoff, M.M. (2004) Incidence of Metastasis to the Ovaries from Nongenital Tract Primary Tumors. Gynecologic Oncology, 93, 87-91. http://dx.doi.org/10.1016/j.ygyno.2003.12.039 